\title{
Use of Imaging for Staging of Early-Stage Breast Cancer in Two Integrated Health Care Systems: Adherence With a Choosing Wisely Recommendation
}

\author{
By Erin E. Hahn, PhD, MPH, Tania Tang, MPH, Janet S. Lee, MS, Corrine Munoz-Plaza, MPH, \\ Joyce O. Adesina, MD, Ernest Shen, PhD, Braden Rowley, BS, Jared L. Maeda, PhD, MPH, \\ David M. Mosen, PhD, MPH, John C. Ruckdeschel, MD, and Michael K. Gould, MD, MS
}

Kaiser Permanente Southern California, Pasadena; Southern California Permanente Medical Group, Los Angeles, CA; Intermountain Healthcare, Salt Lake City, UT; Mid-Atlantic Permanente Research Institute, Rockville, MD; and Kaiser Permanente Center for Health Research, Portland, OR

\begin{abstract}
Purpose: Advanced imaging is commonly used for staging of early-stage breast cancer, despite recommendations against this practice. The objective of this study was to evaluate and compare use of imaging for staging of breast cancer in two integrated health care systems, Kaiser Permanente $(\mathrm{KP})$ and Intermountain Healthcare $(\mathrm{IH})$. We also sought to distinguish whether imaging was routine or used for diagnostic purposes.
\end{abstract}

Methods: We identified patients with stages 0 to IIB breast cancer diagnosed between 2010 and 2012. Using KP and $\mathrm{IH}$ electronic health records, we identified use of computed tomography, positron emission tomography, or bone scintigraphy 30 days before diagnosis to 30 days postsurgery. We performed chart abstraction on a random sample of pa-

\section{Introduction}

Breast cancer is the most commonly diagnosed cancer in women, accounting for approximately $28 \%$ of all new cancer diagnoses, excluding nonmelanoma skin cancers. ${ }^{1}$ Approximately 230,000 new cases of invasive breast cancer were expected to be diagnosed in the United States in 2014. ${ }^{1}$ An additional 62,000 new cases of in situ breast cancer were estimated to be diagnosed. ${ }^{2}$ The majority of these cases, approximately $60 \%$, will be diagnosed at a localized stage. ${ }^{1}$ Use of advanced imaging modalities, including positron emission tomography (PET), computed tomography (CT), and radionuclide bone scans for staging of localized breast cancer is not recommended for asymptomatic women. There is little evidence of benefit (eg, improved detection of metastatic disease or improved survival), ${ }^{3-5}$ and use of advanced imaging may expose patients to unnecessary harms. ${ }^{6,7}$ Evidence-based guidelines from the National Comprehensive Cancer Network (NCCN), as well as guidelines from Cancer Care Ontario and the Alberta Health Services clinical guidelines group, include recommendations against use of advanced imaging for staging of early breast cancer. ${ }^{8-10}$ The 2012 ASCO Top Five list includes "Do not perform PET, CT, and radionuclide bone scans in the staging of early breast cancer at low risk for metastasis" as the tients who received a presurgical imaging test to identify indication.

Results: For the sample of 10,010 patients, mean age at diagnosis was 60 years (range, 22 to 99 years); with 21\% stage 0, 47\% stage I, and $32 \%$ stage II. Overall, $15 \%$ of patients ( $n=1,480)$ received at least one imaging test during the staging window, 15\% at $\mathrm{KP}$ and $14 \%$ at $\mathrm{IH}(P=.5)$. Eight percent of patients received imaging before surgery, and $7 \%$ postsurgery. We found significant intraregional variation in imaging use. Chart abstraction ( $n=129$, $16 \%$ of patients who received presurgical imaging) revealed that $48 \%$ of presurgical imaging was diagnostic.

Conclusion: Use of imaging for staging of low-risk breast cancer was similar in both systems, and slightly lower than has been reported in the literature. Approximately half of imaging tests were ordered in response to a sign or symptom.

third recommendation. ${ }^{7}$ The ASCO Top Five lists, ${ }^{7,11}$ developed as part of the American Board of Internal Medicine Choosing Wisely campaign, emphasize limiting low-value cancer care services. ${ }^{12}$ Choosing Wisely is dedicated to reducing the use of low-value health care services throughout the US health care system. It has received considerable attention in the medical and lay press. ${ }^{13-15}$ A recent survey of primary care and specialty physicians found that physicians exposed to the Choosing Wisely campaign were more likely not to recommend an unnecessary test or procedure than physicians not exposed to the campaign $(62 \% v 45 \%){ }^{16}$

Despite these guideline recommendations, and the recent attention of the Choosing Wisely campaign, it has been shown that use of imaging for staging of early-stage breast cancer is common. ${ }^{17-20}$ In addition, use of advanced imaging modalities has increased over time, particularly in cancer care. ${ }^{21,22}$ The objective of this study was to measure and compare use of imaging for staging of early-stage breast cancer in two large integrated health care systems, Kaiser Permanente (KP) and Intermountain Healthcare (IH). Studies from established integrated systems are valuable as more health care organizations move toward accountable care and medical home models of care delivery, providing insight into how these models may 
influence use of low-value cancer services. We also sought to distinguish whether presurgical imaging was routine or diagnostic (used in response to symptoms or signs).

\section{Methods}

This study was conducted in two integrated systems, KP and IH. We compared use of imaging services between the two systems to assess whether variation in care delivery patterns exists between integrated systems with similar, although not identical, organizational structure. We also examined regional use within each system, to assess whether geographic variation exists within each system, or whether the integrated nature of these systems discourages intrasystem variation. We examined use of PET, CT, and bone scan from 30 days before the recorded diagnosis date in the cancer registry to 30 days after the date of surgery (lumpectomy or mastectomy).

\section{Setting}

$\mathrm{KP}$ is an integrated health care system providing comprehensive health care services to approximately 9 million members. KP operates in eight states and the District of Columbia and is divided into seven regions. For this study, we obtained data from three regions: KP Southern California (KPSC), KP Northwest (KPNW), and KP Mid-Atlantic States (KPMAS). KPSC has the largest membership, with approximately 3.7 million members. Intermountain Healthcare is a regional, not-forprofit integrated health care system in Utah and southern Idaho. It consists of 22 hospitals and nine cancer centers and is divided into four regions: Urban Central Region (UCR), Urban North Region (UNR), Urban South Region (USR), and Southwest Region (SWR). There were approximately one million unique patients with IH hospital visits in 2013, with the largest number in UCR. Patients have a mix of insurance types within these two systems, including commercial insurance, Medicare, and Medicaid. The majority of KP members participate in capitated health plans, whereas $\mathrm{IH}$ is a mixture of capitated and fee-for-service plans. KP is structured as a group model health maintenance organization, and KP medical groups contract exclusively with the KP health plans. IH has a variety of relationships with affiliated providers, with a mix of employed and nonemployed physicians. This study received institutional review board (IRB) approval for all activities, and KPSC acted as lead IRB for all KP study activities (KPSC IRB \#10145; IH IRB\# 1024956).

\section{Identification of Sample}

We identified patients using KP and IH tumor registries. We included female patients diagnosed with stages 0 to IIb breast cancer between January 1, 2010, and December 31, 2012, based on American Joint Committee on Cancer (AJCC) Stage criteria, seventh edition. We restricted the sample to patients who received definitive cancer-directed surgery (Appendix Figure A1, online only). We excluded patients with a history of any cancer diagnosis, excluding nonmelanoma skin cancers. Tumor registry data provided information on date of birth, age at di- agnosis, pathological stage, tumor histology, tumor grade, treatment type(s) (eg, surgical type, chemotherapy, radiation), and treatment dates, with the exception of the KPMAS tumor registry which did not include treatment type.

\section{Data Sources}

We used Current Procedural Terminology codes and International Classification of Diseases (ed 9) to identify PET, CT, and radionuclide bone scans, restricting our choice of imaging services to these three imaging types to reflect the recommendations from the ASCO 2012 Top Five list. We excluded imaging services performed in the emergency department or inpatient setting, as services in this setting are unlikely to be related to staging for clinical early-stage breast cancer, and our intent was to assess usual patterns of care in the oncology setting. Chart abstractions were performed on a stratified random sample of charts by image test type in patients who received presurgical imaging. Our goal was to abstract at least $15 \%$ of patients who received a nonrecommended imaging service, a target chosen as both feasible and likely to provide useful information. Chart abstractors at each site, including research associates, a registered nurse, and an internal medicine physician, were trained with a centralized chart abstraction protocol developed by the study investigators. The abstractors, with supervision and consultation with the site principal investigators and research team, categorized imaging services as diagnostic (used in response to a clinical sign found on physical exam, prior finding, or patient reported symptom) or routine (used in absence of signs or symptoms indicative of potential metastatic disease), based on categories developed by Cooper et al to categorize surveillance services in cancer care. ${ }^{23}$ These categories, although developed to categorize cancer surveillance services, are broadly applicable for capturing clinical indication of services. Categorization of diagnostic or routine was based on the clinician note(s) associated with the imaging service, and documented signs and symptoms were captured in a study database to aid in categorization. A list of relevant signs and symptoms based on the NCCN guideline for invasive breast cancer was used to guide review and abstraction (localized bone pain, abdominal symptoms, abnormal physical exam of abdomen or pelvis, pulmonary symptoms, abnormal test results). ${ }^{9}$ The lack of a definitive statement of a sign or symptom was considered reason to categorize an imaging service as routine.

\section{Data Analysis}

Means, ranges, and percentages were generated as appropriate to summarize patient demographics and cancer disease and treatment characteristics. We calculated the overall number of patients in both systems who received at least one imaging service within the staging window as defined above, as well as by health system and by regions within the systems. We also calculated the overall totals of all three imaging service types (PET, CT, bone scan), as well as by health system and by regions within the systems. We compared results between the two systems and within regions of each system using $\chi^{2}$ and analysis of 
variance tests as appropriate. All analyses were performed using SAS software, version 9.2 (SAS Institute, Cary, NC).

\section{Results}

\section{Demographic, Cancer Diagnosis, and Cancer Treatment Characteristics}

Between 2010 and 2012, there were 10,010 patients with stages 0 to IIb breast cancer diagnosed within the two systems (KP regions: $\mathrm{n}=7,957$; $\mathrm{IH}: \mathrm{n}=2,053$; Table 1 ). The average age at diagnosis within both systems was 60 years (standard deviation $=12$ years). The overall majority of patients were white (60\%), although the race/ethnicity of patients within KP differed significantly by region (KPSC: $51 \%$ white; KPNW: $89 \%$ white; KPMAS: $24 \%$ white, $P<.001)$. Overall, the majority of patients $(47 \%)$ had stage I disease, with small differences between the two systems (KP: $48 \%$ stage I, IH: $43 \%$ stage I, $P<$ .001 ) and within regions. Ductal histology (including infiltrating ductal and infiltrating ductal, mixed) was the most common in both systems (KP: $81 \%$; IH: 67\%, $P<.001$ ), and the majority of patients had a tumor grade of moderately differentiated (KP: 43\%; IH: 41\%, $P<.001$ ). There were statistically significant differences between the systems for cancer treatment. Sixty-four percent of KP patients received a lumpectomy versus $74 \%$ of $\mathrm{IH}$ patients $(P<.001), 32 \%$ of KP patients received chemotherapy versus $35 \%$ of IH patients $(P=.01)$, and $49 \%$ of $\mathrm{KP}$ patients received radiation versus $62 \%$ of $\mathrm{IH}$ patients $(P<$ $.001)$. There was also statistically significant variation in treatment within the regions as shown in Table 1 . Note that treatment characteristics do not include data from the KPMAS region; denominators for KP were adjusted accordingly.

\section{Use of Imaging for Staging}

Overall, $15 \%$ of patients (1,480 patients) received at least one imaging test (Table 2). Eight percent of patients received at least one imaging service in the presurgical period, and $7 \%$ in the postsurgical period. The most commonly used imaging modality was CT scan, accounting for $73 \%$ of all imaging tests. We found statistically insignificant differences between the two systems: $15 \%$ of KP patients received at least one imaging service versus $14 \%$ at $\mathrm{IH}(P=.5)$. There were statistically significant differences within regions for use of imaging services. Within the three KP regions, $15 \%$ of KPSC patients received at least one imaging service, $10 \%$ of KPNW patients received at least one, and $21 \%$ of KPMAS patients received at least one $(P<$ .001). Within the $4 \mathrm{IH}$ regions, $28 \%$ of UNR patients received at least one, versus $18 \%$ in USR, $16 \%$ in SWR, and 6\% in UCR $(P<.001)$.

\section{Clinical Indication}

Chart abstraction $(\mathrm{n}=129 ; 16 \%$ of patients who received presurgical imaging) revealed that close to half (48\%) of all imaging tests were performed in response to a clinical sign or patient-reported symptom (Table 3). For imaging services categorized as diagnostic, common reasons included response to abnormal laboratory and imaging findings (25\%), palpable masses or noted axillary lymphadenopathy (30\%), abdominal pain $(15 \%)$, chest pain $(10 \%)$, other patient-reported pain $(10 \%)$, and unexplained weight loss (10\%). Overall, CT scans were most commonly categorized as diagnostic (70\%), whereas PET scans were most commonly categorized as routine (96\%). Bone scans were divided equally between diagnostic and routine. We found differences between the two systems for diagnostic versus routine use: $55 \%$ of imaging services at $\mathrm{KP}$ were performed in response to a sign or symptom versus $33 \%$ at IH. We also found differences within the regions. Within the three $\mathrm{KP}$ regions, $78 \%$ of imaging services in the KPSC region were performed in response to a sign or symptom, versus $53 \%$ in KPNW and 37\% in KPMAS. Within the four IH regions, $40 \%$ of imaging services in the UCR and UNR regions were performed in response to a sign or symptom, versus 30\% in USR and $20 \%$ in SWR (data not shown).

\section{Discussion}

The recent ASCO Top Five lists have drawn attention to use of low-value cancer care services, including use of imaging for staging of early breast cancer. We examined use of PET, CT, and radionuclide bone scan for staging within two large integrated health care systems. Overall, $15 \%$ of patients with breast cancer received a PET, CT, or radionuclide bone scan during the staging window, including $15 \%$ at $\mathrm{KP}$ and $14 \%$ at $\mathrm{IH}$. This is somewhat less than has been reported in the literature. A recent study using SEER data linked with Medicare claims showed that $19 \%$ of patients with breast cancer diagnosed between 1992 and 2005 received preoperative imaging services. ${ }^{17}$ Other studies using claims data have shown that $15 \%$ to $42 \%$ of patients with breast cancer received imaging services for staging. ${ }^{19,20,24}$ Patient demographic and treatment characteristics (Table 1) vary significantly between the systems, particularly the race/ethnicity of patients, yet overall use of imaging for staging is quite similar at the system level. However, we found statistically significant variation within regions of each system, suggesting that imaging use may be driven by patient and provider factors distinct from the integrated setting.

Importantly, we were able to categorize use of imaging services as diagnostic or routine. Studies examining patterns of use commonly rely on data from administrative claims databases. ${ }^{17,19}$ These data sources do not provide information on diagnostic versus routine use (eg, clinical indication), a significant limitation. We found that approximately half of all imaging services in the presurgical period (48\%) were used in response to a sign or symptom. For those imaging services categorized as diagnostic, clinical documentation in the electronic health record (EHR) included signs and symptoms that may be associated with metastatic disease, such as patient-reported pain and palpable masses. In these situations, use of imaging for staging in response to signs or symptoms represents guidelineconcordant care. ${ }^{9}$ A recent survey showed that medical, radiation, and surgical oncologists consider clinical signs and symptoms to be important factors when deciding whether or not to use imaging for staging of early-stage breast cancer. ${ }^{25}$ Our results illustrate the importance of capturing clinical indi- 


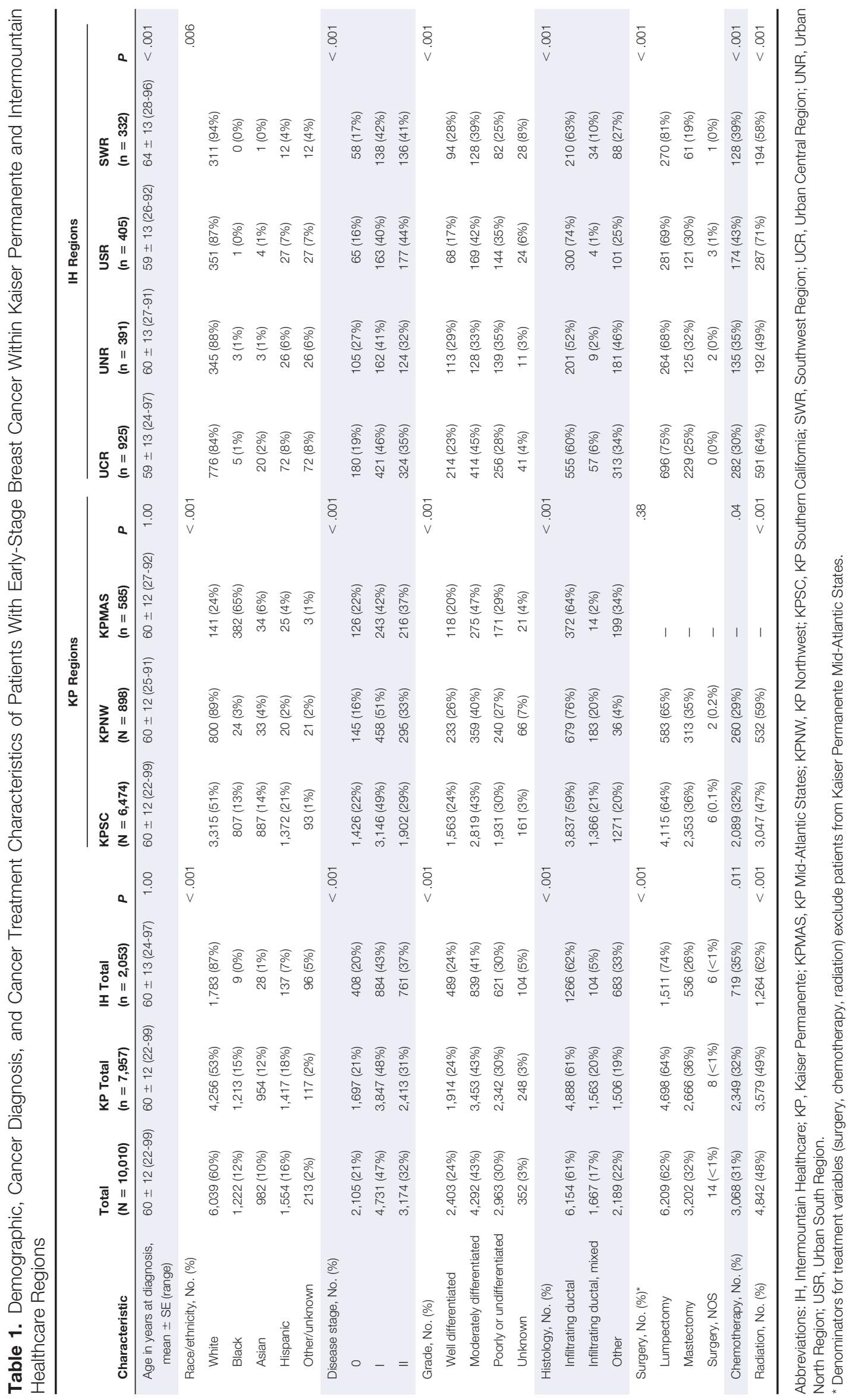


Table 2. Use of Imaging Services 30 Days Before Date of Diagnosis to 30 Days Post-Surgery for Patients With Early-Stage Breast Cancer Within Kaiser Permanente and Intermountain Healthcare Regions

\begin{tabular}{|c|c|c|c|c|c|c|c|c|c|c|c|c|c|}
\hline Imaging Services & $\begin{array}{l}\text { Total } \\
(\mathrm{N}=10,010)\end{array}$ & $\begin{array}{l}\text { KP Total } \\
\text { (n = 7,957) }\end{array}$ & $\begin{array}{l}\text { IH Total } \\
(n=2,053)\end{array}$ & $P$ & \multicolumn{4}{|c|}{ KP Regions } & \multicolumn{5}{|c|}{ IH Regions } \\
\hline Any imaging, №. (\%)† & 1,480 (15\%) & 1,186 (15\%) & $294(14 \%)$ & .5 & $975(15 \%)$ & $88(10 \%)$ & $123(21 \%)$ & $<.001$ & $60(6 \%)$ & $110(28 \%)$ & $71(18 \%)$ & $53(16 \%)$ & $<.001$ \\
\hline CT $\ddagger$ & $1,196(12 \%)$ & 1,002 (13\%) & $194(9 \%)$ & $<.001$ & $857(13 \%)$ & $52(6 \%)$ & $93(16 \%)$ & $<.001$ & $37(4 \%)$ & 95 (24\%) & $36(9 \%)$ & $26(8 \%)$ & $<.001$ \\
\hline PET & $224(2 \%)$ & $124(2 \%)$ & $100(5 \%)$ & $<.001$ & $83(1 \%)$ & $24(3 \%)$ & $17(3 \%)$ & $<.001$ & $23(2 \%)$ & $14(4 \%)$ & $34(8 \%)$ & $29(9 \%)$ & $<.001$ \\
\hline CT & 2,174 & 1,980 & 194 & - & 1,814 & 59 & 107 & - & 37 & 95 & 36 & 26 & - \\
\hline PET & 235 & 135 & 100 & - & 88 & 28 & 19 & - & 23 & 14 & 34 & 29 & - \\
\hline Bone & 566 & 530 & 36 & - & 479 & 20 & 31 & - & 8 & 17 & 6 & 5 & - \\
\hline \multicolumn{14}{|c|}{30 Days Before Date of Diagnosis to Initial Surgery } \\
\hline \multicolumn{14}{|c|}{30 Days Post-Surgery } \\
\hline Any imaging, №. (\%)† & 739 (7\%) & $565(7 \%)$ & $174(8 \%)$ & $<.05$ & $451(7 \%)$ & $37(4 \%)$ & $77(13 \%)$ & $<.001$ & $28(3 \%)$ & $80(20 \%)$ & $39(10 \%)$ & $27(8 \%)$ & $<.001$ \\
\hline CT‡ & $574(6 \%)$ & $442(6 \%)$ & $132(6 \%)$ & .1 & $364(6 \%)$ & $20(2 \%)$ & $58(10 \%)$ & $<.001$ & $21(2 \%)$ & $76(19 \%)$ & $20(5 \%)$ & $15(5 \%)$ & $<.001$ \\
\hline PET & $82(1 \%)$ & $41(1 \%)$ & $41(2 \%)$ & $<.001$ & $30(0 \%)$ & $7(1 \%)$ & $4(1 \%)$ & .4 & $7(1 \%)$ & $2(1 \%)$ & $18(4 \%)$ & $14(4 \%)$ & $<.001$ \\
\hline Bone & $263(3 \%)$ & $250(3 \%)$ & $13(1 \%)$ & $<.001$ & $216(3 \%)$ & $10(1 \%)$ & $24(4 \%)$ & $<.001$ & $3(0 \%)$ & $8(2 \%)$ & $2(0 \%)$ & $0(0 \%)$ & $<.01$ \\
\hline
\end{tabular}

Abbreviations: $\mathbb{H}$, Intermountain Healthcare; KP, Kaiser Permanente; KPMAS, KP Mid-Atlantic States; KPNW, KP Northwest; KPSC, KP Southern California; SWR, Southwest Region; UCR, Urban Central Region; UNR, Urban North Region; USR, Urban South Region.

${ }^{*} P$ value is derived from chi-squared test.

† The value displayed for 'Any Imaging' is defined as the number of patients with at least one imaging test performed. The number of patients with any imaging test is less than the sum of patients with CT, PET, or bone scan due to multiple tests performed on some patients.

$\ddagger$ The number of unique patients with at least one imaging test is displayed for CT, PET, and bone scan.

cation when measuring adherence to guideline-recommended care. This is critical when assessing use of low-value services as described by the Choosing Wisely campaign. Failure to do so may result in biased results, leading to efforts to change practice patterns inappropriately. In addition, it is important to acknowledge that there is debate about how to approach patients with breast cancer who present with large cancers or clinically palpable nodes. Fuster et al demonstrated that PET CT led to a change in initial staging in $42 \%$ of women with large $(>3 \mathrm{~cm}$ ) cancers, ${ }^{26}$ and Groheux et al showed that stage was modified by
PET imaging $16 \%$ of the time in women with stage IIb breast cancer. ${ }^{27}$ Within our data, 59\% of PET scans ordered during the staging window were in patients with stage IIb breast cancer, $70 \%$ within $\mathrm{KP}$ and $49 \%$ within IH. It may not be unreasonable to consider PET imaging in stage IIb patients.

This study has several strengths. We examined a large sample from several geographically distinct regions, and supplemented analysis of structured data from EHRs with in-depth chart reviews. We examined data for patients diagnosed between January 1, 2010, and December 31, 2012. These data represent

Table 3. Chart Abstraction Results for Routine Versus Diagnostic (clinically indicated) Use of Imaging Services for Staging of Patients With Early-Stage Breast Cancer Within Kaiser Permanente and Intermountain Healthcare Regions

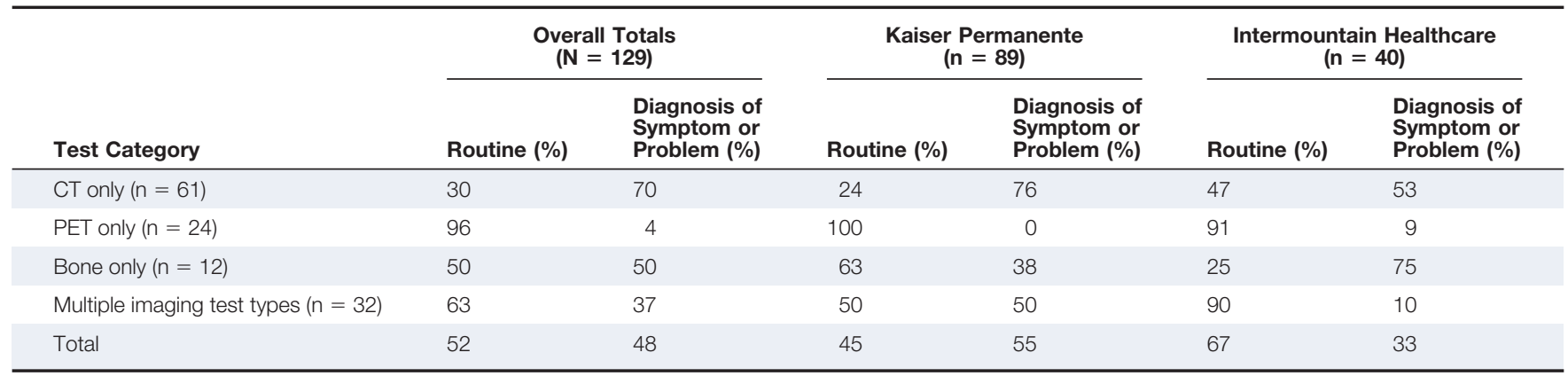

NOTE. Kaiser Permanente regions include only Southern California, Northwest, and Mid-Atlantic States.

Abbreviations: $\mathrm{CT}$, computed tomography; PET, positron emissions tomography. 
recent clinical activities and provide an up-to-date synopsis of imaging use patterns during staging for patients with breast cancer within these two health care systems. An additional strength is that we had access to complete patient data from the integrated model of both health care systems. Given that both systems provide comprehensive health care services, it is unlikely that patients sought care outside of $\mathrm{KP}$ or $\mathrm{IH}$ for additional clinical services. In addition, we were conservative in our categorization of services as diagnostic or routine. Lack of a definitive statement about a symptom or sign in the associated EHR clinician notes was considered reason to categorize the service as routine. This reduced the likelihood of overestimating the number of imaging services used for diagnostic purposes.

This study also has several limitations. We included only the nonrecommended imaging services included in the ASCO 2012 Top Five recommendation: PET, CT, and radionuclide bone scan. We did not look at chest $\mathrm{x}$-ray, breast magnetic resonance imaging, or other imaging services. In addition, while we had data from the entire IH system, we included data from only three of seven KP regions. It is possible that data from other $\mathrm{KP}$ regions would produce different results. However, we were able to include data from KPSC, which is the largest KP region based on membership. An additional limitation is the small number of chart abstractions we conducted on presurgical use of imaging, $16 \%$ of those who received an imaging test $(\mathrm{N}=129)$. However, our results did show clear divisions on diagnostic versus routine staging use of imaging. For example, PET scans were consistently categorized as routine staging; CT scans consistently categorized as diagnostic; and bone scans were consistently categorized as half staging, half diagnostic. This consistency in our results provides credibility. Finally, the generalizability of our results is limited given that these data represent patterns of use in two large, integrated health care systems. However, the US health care system is moving toward models of care that encourage integration and accountability. The results of this research are directly relevant to the growing number of these models of care delivery, such as patientcentered medical homes and accountable care organizations. In addition, results from this study provide a comparison to studies using large datasets from multiple sources, such as SEER-Medicare data.

In conclusion, we found similar overall use of imaging for staging of early-stage breast cancer in two large, integrated

\section{References}

1. American Cancer Society: Cancer Facts \& Figures 2014. Atlanta, GA, American Cancer Society

2. DeSantis C, Ma J, Bryan L, et al: Breast cancer statistics. CA Cancer J Clin 64:52-62, 2014

3. Puglisi F, Follador A, Minisini AM, et al: Baseline staging tests after a new diagnosis of breast cancer: Further evidence of their limited indications. Ann Oncol 16:263-266, 2005

4. Brennan ME, Houssami N: Evaluation of the evidence on staging imaging for detection of asymptomatic distant metastases in newly diagnosed breast cancer. Breast 21:112-123, 2012

5. Al-Husaini H, Amir E, Fitzgerald B, et al: Prevalence of overt metastases in locally advanced breast cancer. Clin Oncol (R Coll Radiol) 20:340-344, 2008

6. Smith-Bindman R, Lipson J, Marcus R, et al: Radiation dose associated with common computed tomography examinations and the. Arch Intern Med 169: 2078-2086, 2009 health care systems, although each system has considerable intraregional variation. Approximately half of presurgical imaging services were used in response to a clinical sign or patientreported symptom. Delivering high-quality cancer care, which includes limiting use of low-value cancer care services, is imperative given the rising costs of cancer care and the increasing number of patients with cancer and survivors. ${ }^{28}$ However, in examining use of guideline-concordant care and implementing strategies to reduce unnecessary services, we must recognize the need for flexibility and physician autonomy.

\section{Acknowledgment}

Supported by the Center for Safety and Effectiveness Research (CESR), No. CESR 201308. CESR facilitates research conducted by the research programs in each of the seven Kaiser Permanente regional entities (Colorado, Hawaii, Georgia, Mid-Atlantic States Nirginia, Maryland, District of Columbia], Northern California, Northwest [Oregon, Washington] and Southern California) that compose the Kaiser Permanente integrated health care organization, and facilitates comparative systems research. Previously presented as an oral abstract and poster at the American Society of Clinical Oncology Quality Care Symposium, Boston, MA, October 18-20, 2014.

\section{Authors' Disclosures of Potential Conflicts of Interest}

Disclosures provided by the authors are available with this article at jop.ascopubs.org.

\section{Author Contributions}

Conception and design: Erin E. Hahn, Tania Tang, Jared L. Maeda, David M. Mosen, John C. Ruckdeshel, Michael K. Gould

Financial support: Michael K. Gould

Administrative support: Tania Tang

Collection and assembly of data: Tania Tang, Corrine Munoz-Plaza, John C. Ruckdeshel

Data analysis and interpretation: Tania Tang, Janet S. Lee, Corrine Munoz-Plaza, Joyce O. Adesina, Ernest Shen, Braden Rowley, Jared L. Maeda, David M. Mosen, John C. Ruckdeshel, Michael K. Gould

Manuscript writing: All authors

Final approval of manuscript: All authors

Corresponding author: Erin E. Hahn, PhD, MPH, Kaiser Permanente Southern California, Department of Research and Evaluation, 100 S Los Robles Ave, Pasadena, CA 91101; e-mail: Erin.E.Hahn@kp.org.

DOI: 10.1200/JOP.2014.002998; published online ahead of print at jop.ascopubs.org on April 21, 2015.

7. Schnipper LE, Smith TJ, Raghavan D, et al: American Society of Clinical Oncology identifies five key opportunities to improve care and reduce costs: The top five list for oncology. J Clin Oncol 30:1715-1724, 2012

8. Myers RE, Johnston M, Pritchard K, et al: Baseline staging tests in primary breast cancer: A practice guideline. CMAJ 164:1439-1444, 2001

9. National Comprehensive Cancer Network: NCCN Guidelines: Invasive Breast Cancer BINV-1. 2012. www.nccn.org/professionals/physician_gls/pdf/breast. pdf

10. Alberta Health Services Clinical Practice Guideline, version 2, effective date July 2012. www.albertahealthservices.ca/hp/if-hp-cancer-guide-br012-staginginvestigations.pdf

11. Schnipper LE, Lyman GH, Blayney DW, et al: American Society of Clinical Oncology 2013 top five list in oncology. J Clin Oncol 31:4362-4370, 2013

12. Choosing Wisely: An initiative of the ABIM Foundation. mww.choosingwisely.org 
13. Rabin R: Doctor panels recommend fewer tests for patients. New York Times 2012. www.nytimes.com/2012/04/04/health/doctor-panels-urge-fewer-routinetests.html?_r=0

14. Morden NE, Colla CH, Sequist TD, et al: Choosing wisely-The politics and economics of labeling low-value services. N Engl J Med 370:589-592, 2014

15. Consumer Reports Health: Choosing Wisely: How to Avoid Unnexessary Tests and Treatment. 2014. www.consumerreports.org/cro/2012/04/choosingwisely-how-to-avoid-unnecessary-tests-and-treatments/index.htm

16. PerryUndum Reserch/Communications: Unnecessary Tests and Procedures in the Health Care System. www.choosingwisely.org/wp-content/uploads/2014/ 04/042814_Final-Choosing-Wisely-Survey-Report.pdf2014

17. Crivello ML, Ruth K, Sigurdson ER, et al: Advanced imaging modalities in early stage breast cancer: Preoperative use in the United States Medicare population. Ann Surg Oncol 20:102-110, 2013

18. McWhirter $\mathrm{E}$, Yogendran $\mathrm{G}$, Wright $\mathrm{F}$, et al: Baseline radiological staging in primary breast cancer: Impact of educational interventions on adherence to published guidelines. J Eval Clin Pract 13:647-650, 2007

19. Barcenas C, Niu J, Valero V, et al: The use of imaging and tumor markers in the staging of patients age $<65$ years with early-stage breast cancer (Abstract P3-06-02). Cancer Res 73, 2013 (suppl 24; abstr P30602)

20. Dillman RO, Chico S: Radiologic tests after a new diagnosis of breast cancer. Eff Clin Pract 3:1-6, 2000
21. Dinan MA, Curtis LH, Hammill BG, et al: Changes in the use and costs of diagnostic imaging among Medicare beneficiaries with cancer, 1999-2006. JAMA 303:1625-1631, 2010

22. Smith-Bindman R, Miglioretti DL, Larson EB: Rising use of diagnostic medical imaging in a large integrated health system. Health Aff (Millwood) 27:1491-1502, 2008

23. Cooper GS, Johnson CC, Lamerato L, et al: Use of guideline recommended follow-up care in cancer survivors: Routine or diagnostic indications? Medical Care 44:590-594, 2006

24. Gold LS, Buist DS, Loggers ET, et al: Advanced diagnostic breast cancer imaging: Variation and patterns of care in Washington state. J Oncol Pract 9:e194-e202, 2013

25. Simos D, Hutton B, Graham I, et al: Staging imaging for metastatic disease in patients with early-stage breast cancer: What do physicians think of the ASCO top-5 recommendation? J Clin Oncol 32:433s, 2014 (suppl; abstr 6596)

26. Fuster D, Duch J, Paredes $P$, et al: Preoperative staging of large primary breast cancer with [18F]fluorodeoxyglucose positron emission tomography/computed tomography compared with conventional imaging procedures. J Clin Oncol 26:4746-4751, 2008

27. Groheux D, Moretti JL, Baillet G, et al: Effect of (18)F-FDG PET/CT imaging in patients with clinical stage II and III breast cancer. Int J Radiat Oncol Biol Phys 71:695-704, 2008

28. Levit L, Balogh E, Nass S, et al: Delivering High-Quality Cancer Care: Charting a New Course for a System in Crisis. Washington, DC, National Academies Press, 2013 


\section{AUTHORS' DISCLOSURES OF POTENTIAL CONFLICTS OF INTEREST}

Use of Imaging for Staging of Early-Stage Breast Cancer in Two Integrated Health Care Systems: Adherence With a Choosing Wisely Recommendation

The following represents disclosure information provided by authors of this manuscript. All relationships are considered compensated. Relationships are self-held unless noted. I = Immediate Family Member, Inst = My Institution. Relationships may not relate to the subject matter of this manuscript. For more information about ASCO's conflict of interest policy, please refer to www.asco.org/rwc or jop.ascopubs.org/site/misc/ifc.xhtml.

\section{Erin E Hahn}

Employment: Southern California Permanente Medical Group

\section{Tania Tang}

Employment: Amgen (I)

Stock or Other Ownership: Amgen (I)

Travel, Accommodations, Expenses: Amgen (I)

\section{Janet S Lee}

Employment: Southern California Permanente Medical Group

\section{Corrine Munoz-Plaza}

Employment: Southern California Permanente Medical Group

\section{Joyce O. Adesina}

No relationship to disclose

\section{Ernest Shen}

No relationship to disclose

\section{Braden Rowley}

Employment: PRA Health Sciences

\section{Jared L. Maeda}

Employment: Mid-Atlantic Permanente Medical Group

David M. Mosen

No relationship to disclose

\section{John C. Ruckdeshel}

No relationship to disclose

Michael K. Gould

Employment: Southern California Permanente Medical Group Honoraria: UpToDate

Research Funding: Archimedes (Inst) 


\section{Appendix}

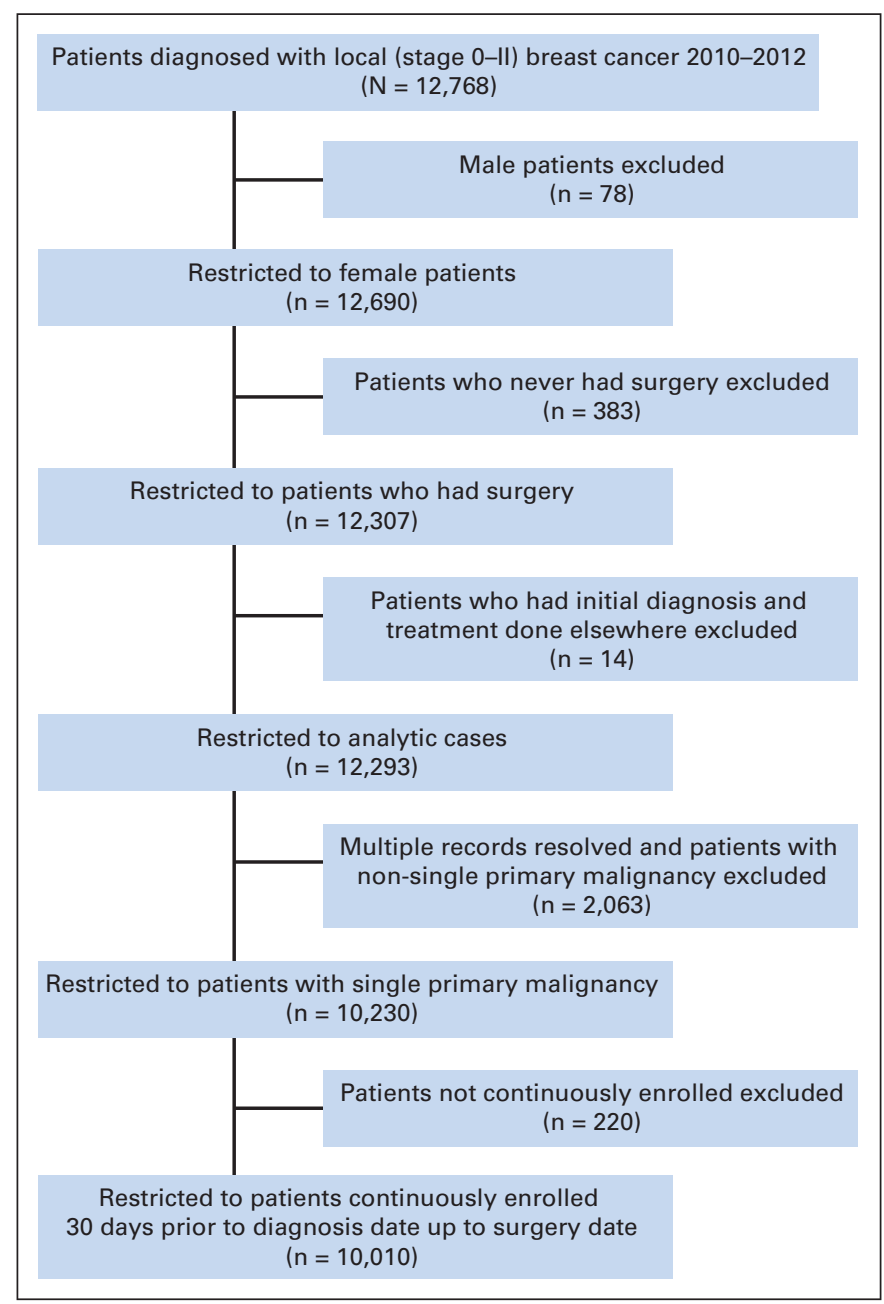

Figure A1. Cohort flow diagram for patients with early-stage (stage 0-llb) breast cancer diagnosed between January 1, 2010, and December 31, 2012 identified within Kaiser Permanente (Southern California, Northwest, and Mid-Atlantic States regions) and Intermountain Healthcare; N = 10,010. 